\title{
On the critical thickness and wavelength of a condensing thin liquid film in a binary vapor mixture system
}

\author{
Kentaro Kanatani* \\ Faculty of Engineering, Yokohama National University, \\ 79-5 Tokiwadai, Hodogaya, Yokohama 240-8501, Japan
}

\begin{abstract}
We study the linear stability of a condensing thin liquid film of a binary vapor mixture by solving directly the bulk equations of the gas phase. The boundary layer of a finite thickness is introduced above the liquid film, within which the variables are disturbed. The dynamics of the liquid film is described by the long-wave equation. The neutral stability condition predicts the existence of a critical thickness below which a flat film is stable due to the mass gain effect. However, if we consider the thickening of the liquid film by condensation, the relative neutral stability can be defined such that the growth rate of a disturbance is equal to that of the basic film thickness. The critical thickness and wavenumber obtained from the relative neutral stability condition significantly change from the original ones. Employing the asymptotic analysis for large wavenumbers, the critical thickness and wavelength are numerically calculated for the water-ethanol system. Their dependence on the boundary layer thickness, temperature and ambient vapor concentration is investigated. The critical wavelength obtained from our theory has the same trend in the temperature and concentration as the initial drop distance observed in the experiment.
\end{abstract}

Keywords: Marangoni dropwise condensation, Binary mixture, Stability analysis, Thin liquid film

\footnotetext{
*Tel. / Fax: +81-45-339-3897.

Email address: kentaro@ynu.ac.jp (Kentaro Kanatani)
} 


\section{Introduction}

Since the first appearance of Marangoni dropwise condensation [1], a number of experimental studies have been devoted to condensation of binary vapor mixtures to observe nonfilmwise condensation $[2,3,4,5]$. In contrast, there are few theoretical works on Marangoni dropwise condensation. Among them, Hijikata et al. [6] investigated the stability of a flat condensate of a binary vapor mixture. Recently, we modeled the dynamics of a condensate liquid film of a binary vapor mixture using the long-wave approximation and the linear stability analysis was carried out based on this model [7]. In both studies the vapor concentration gradient at the liquidvapor interface in the vertical direction is assumed to be proportional to the concentration difference between the ambient vapor and interface, which is an analogue of well-known Newton's law of cooling for heat transfer. However, if a flat condensate is disturbed the vapor concentration also fluctuates along the liquid-vapor interface. This fluctuation makes the vapor concentration diffuse along the interface. The diffusion of the vapor concentration was disregarded in the above models [6, 7].

In the modeling of an evaporating liquid film, Sultan et al. [8, 9] took into account static diffusion of the vapor in the long-wave equation describing the spatio-temporal evolution of the film thickness $[10,11]$ by solving the Laplace equation for the vapor concentration. Recently, we proposed a model including advective effects due to the evaporation flow of the gas [12]. In this model, a rather ad hoc model is applied to the vertical diffusion term of the mass transport equation.

In this work, we study the linear stability of a condensing thin liquid film of a binary vapor mixture by solving directly the bulk equations of the gas phase without applying any model to the vapor concentration. As in the previous studies [7, 12, 13], the boundary layer of a finite thickness is introduced above the liquid layer, within which the variables are disturbed. Then the disturbances in the gas phase are coupled with that of the film thickness of the condensate, which is described by the long-wave equation $[7,11]$. Here for simplicity the frozen-time approach is adopted, where the linear stability analysis is carried out against the 'hypothetically motionless' interface at each instance or film thickness (of course in reality the interface is moving owing to condensation). As a result, the neutral stability condition is obtained by setting the growth rate of the disturbances to zero. Since the effect of mass gain is stabilizing, there is a critical thickness below which a flat 
film is stable. However, if the condensate is sufficiently thin (about $1 \mu \mathrm{m}$ ), the temporal evolution of the average film thickness becomes comparable to that of the disturbances. In this case, it is insufficient to address only the growth rate of the disturbances: we have to consider the evolution of the ratio of the amplitude of the disturbance of the film thickness to the average film thickness. If this ratio temporarily increases (decreases), it follows that the disturbance grows (decays) relatively to the average film thickening. Thus the relative neutral stability can be defined such that the time derivative of this ratio vanishes. We will show that this definition of the neutral stability significantly changes the critical thickness and wavenumber compared to the original ones. The dependence of the critical thickness and wavelength on the boundary layer thickness, temperature and ambient vapor concentration is investigated. A comparison is made with the experimental result for the water-ethanol system [5].

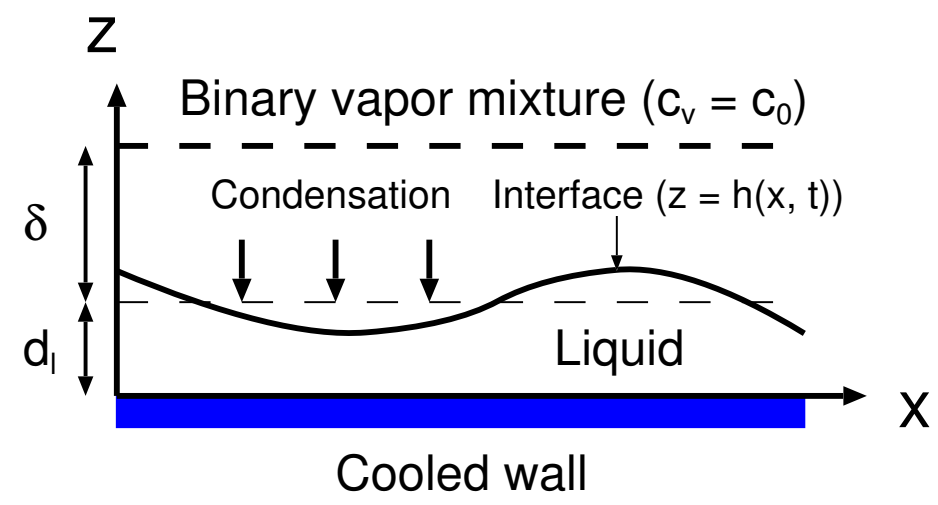

Figure 1: Sketch of a condensate liquid film of a binary vapor mixture on a cooled horizontal surface.

\section{Formulation}

We consider a two-dimensional condensate liquid film of a binary vapor mixture on a uniformly-cooled horizontal wall, as depicted in Fig. 1. The $x$ axis coincides with the liquid-solid boundary and the $z$-axis points vertically upward to the wall. The mass fraction of the surrounding vapor is fixed to $c_{0}$ at some distance $\delta$ from the average height of the liquid-gas interface; 
here $\delta$ is assumed to be much larger than the thickness of the film $h$ so that the former is never affected by the variation of the latter. We scale lengths, time, velocities and pressure of the gas phase by $d_{l}, d_{l}^{2} / \nu_{l}, \nu_{l} / d_{l}$ and $\rho_{v} \nu_{l}^{2} / d_{l}^{2}$, respectively, where $d_{l}, \nu_{l}$, and $\rho_{v}$ are the initial film thickness, the kinematic viscosity of the liquid, and the vapor density, respectively. The dimensionless governing equations in the gas phase are the continuity, Navier-Stokes, and mass transport equations:

$$
\begin{aligned}
& \nabla \cdot \boldsymbol{v}_{v}=0 \\
& \partial_{t} \boldsymbol{v}_{v}+\boldsymbol{v}_{v} \cdot \nabla \boldsymbol{v}_{v}=-\nabla p_{v}+\nu \nabla^{2} \boldsymbol{v}_{v}-G \boldsymbol{e}_{z}, \\
& \Sigma_{v}\left(\partial_{t} c_{v}+\boldsymbol{v}_{v} \cdot \nabla c_{v}\right)=\nu \nabla^{2} c_{v},
\end{aligned}
$$

where $\boldsymbol{v}_{v}=\left(u_{v}, w_{v}\right), p_{v}$, and $c_{v}$ are the velocity, pressure, and mass concentration of the component having a lower boiling point in the gas, respectively. The differential operator is $\nabla \equiv\left(\partial_{x}, \partial_{z}\right)$. The symbols $\nu=\nu_{v} / \nu_{l}$, $G=g d_{l}^{3} / \nu_{l}^{2}$, and $\Sigma_{v}=\nu_{v} / D_{v}$ denote the kinematic viscosity ratio, the Galileo number, and the Schmidt number of the vapor, where $\nu_{v}, g$, and $D_{v}$ are the kinematic viscosity, the gravitational acceleration, and mass diffusivity of the gas phase, and $\boldsymbol{e}_{z}$ is the unit vector in the $z$ direction. In Eqs. (1a) and (1b) we have neglected the concentration dependence of $\rho_{v}$ and $\nu_{v}$ considering a small concentration difference between the ambient vapor and liquid-gas interface or a small condensation rate. In Eq. (1b) buoyancy has been neglected; however, we shall discuss its influence on the stability of the liquid film in Appendix A. In addition we have neglected also thermo-diffusion and diffusion-thermo effects.

For the liquid phase, the following long-wave equation for the film thickness $h(x, t)$ and mass transport equation hold [11]:

$$
\begin{gathered}
\partial_{t} h=\partial_{x}\left\{\frac{h^{3}}{3}\left(G \partial_{x} h-S \partial_{x x x} h\right)-\frac{h^{2}}{2} M a \partial_{x} T_{I}\right\}+\Gamma J, \\
\Sigma_{l}\left(\partial_{t} c_{l}+\boldsymbol{v}_{l} \cdot \nabla c_{l}\right)=\nabla^{2} c_{l},
\end{gathered}
$$

where the subscripts $l$ and $I$ refer to the liquid and interfacial counterparts of the same symbol. The dimensionless numbers are defined by

$$
S=\frac{\sigma d_{l}}{\eta_{l} \nu_{l}}, \quad M a=-\frac{d_{l} L \rho_{v} D_{v}}{\eta_{l} \nu_{l} \lambda_{l}}\left(\frac{\partial \sigma}{\partial T}+\frac{\partial \sigma}{\partial c_{l}} \frac{\partial c_{l}}{\partial T}\right), \quad \Gamma=\frac{\rho_{v} D_{v}}{\eta_{l}},
$$

which characterize the effects of surface tension, thermo- and soluto-capillarity, and mass gain, respectively. Here, $\sigma$ is the surface tension, $\eta_{l}$ is the dynamic 
viscosity of the liquid, $L$ is the latent heat and $\lambda_{l}$ is the thermal conductivity of the liquid. The condensation mass flux $J(x, t)$ and interface temperature $T_{I}(x, t)$ have been nondimensionalized by $\rho_{v} D_{v} / d_{l}$ and $L \rho_{v} D_{v} / \lambda_{l}$. We assume the linear temperature profile across the liquid film, which is a natural consequence of the long-wave approximation (see for example Ref. [11]). Since the heat flux in the liquid balances with the latent heat (the heat flux in the gas is negligible), the interface temperature can be written as

$$
T_{I}=J h
$$

where the wall temperature has been assumed to be constant and set to 0 . For simplicity, we shall neglect the gravitational term in Eq. (1d) by setting $G=0$, which is normally justified for very thin films (say a few micrometers' thickness).

At the top of the gas boundary layer $\left(z=1+\Delta ; \Delta=\delta / d_{l}\right)$, we impose the stress-free and constant pressure and concentration boundary conditions,

$$
\partial_{z} u_{v}+\partial_{x} w_{v}=0, \quad p_{v}=0, \quad c_{v}=c_{0},
$$

where the dimensionless pressure has been set to 0 there. At the wall $(z=0)$, the reflecting concentration boundary condition holds

$$
\partial_{z} c_{l}=0 .
$$

At the liquid-gas interface $(z=h(x, t))$, the conservation laws of the total mass and that of one component and the continuity of the tangential velocity along the interface read

$$
\begin{aligned}
-\nu J & =\Sigma_{v} \boldsymbol{v}_{v} \cdot \boldsymbol{n}, \\
\nabla c_{l} \cdot \boldsymbol{n}+c_{l} J & =\nabla c_{v} \cdot \boldsymbol{n}+c_{v} J . \\
\boldsymbol{v}_{l} \cdot \boldsymbol{t} & =\boldsymbol{v}_{v} \cdot \boldsymbol{t} .
\end{aligned}
$$

Here $\boldsymbol{n}=\left(-\partial_{x} h, 1\right) / \sqrt{1+\left(\partial_{x} h\right)^{2}}$ and $\boldsymbol{t}=\left(1, \partial_{x} h\right) / \sqrt{1+\left(\partial_{x} h\right)^{2}}$ are the unit vectors normal and tangent to the interface. In Eq. (4c) we have neglected the interface velocity compared to the vertical vapor velocity. As in our previous study [7], we employ the linearized local thermodynamic equilibrium conditions at the interface:

$$
\begin{aligned}
c_{l I} & =c_{l r}+c_{l}^{\prime}\left(T_{I}-T_{r}\right), \\
c_{v I} & =c_{v r}+c_{v}^{\prime}\left(T_{I}-T_{r}\right),
\end{aligned}
$$


where $c_{l r}$ and $c_{v r}$ are the liquid and vapor concentrations in the thermodynamic equilibrium state at the reference temperature $T_{r}$, and $c_{l}^{\prime}$ and $c_{v}^{\prime}$ are the temperature derivatives of the liquid and vapor concentrations. Although the reference temperature was set to the wall one in our previous study [7], it will be found to be more convenient to fix the initial interface temperature rather than the wall one in the present analysis. For this reason in this study we shall set the reference temperature to the initial interface one.

\section{Basic state and linearized equations}

In the basic state all the variables do not depend on the horizontal coordinate $x$ and there is no mean flow in the horizontal direction. Following Ref. [13] the quasi-stationarity in the gas phase is assumed, where we neglect the temporal derivative terms in Eqs. (1b) and (1c) in obtaining the velocity and concentration fields of the basic state. From the continuity equation (1a) the vertical component of the basic gas velocity is constant, whereas the horizontal one is zero by definition. Since the liquid is static in the basic state the liquid concentration field is also uniform from Eqs. (1e) and (4b). The boundary conditions $(4 \mathrm{c})$ and $(4 \mathrm{~d})$ are reduced in the basic state to

$$
\begin{gathered}
-\nu \bar{J}=\Sigma \bar{w}_{v} \\
\left(\bar{c}_{v I}-\bar{c}_{l I}\right) \bar{J}+\bar{c}_{v}^{\prime}(1)=0,
\end{gathered}
$$

where an overbar refers to the basic state quantity. The basic condensation mass flux and vapor concentration distribution are given by (see Ref. [7])

$$
\begin{gathered}
\bar{J}=\frac{1}{\Delta} \log \left(\frac{\bar{c}_{v I}-\bar{c}_{l I}}{c_{0}-\bar{c}_{l I}}\right) \\
\bar{c}_{v}(z)=c_{0}+\left(\bar{c}_{v I}-c_{0}\right) \frac{\mathrm{e}^{\bar{J}(1+\Delta-z)}-1}{\mathrm{e}^{\bar{J} \Delta}-1}=c_{0}+\left(c_{0}-\bar{c}_{l I}\right)\left\{\mathrm{e}^{\bar{J}(1+\Delta-z)}-1\right\},
\end{gathered}
$$

which are obtained by solving the one-dimensional stationary convectiondiffusion equation in the vertical direction with the boundary conditions (4a) and (5).

The solution of Eqs. (1) and boundary conditions (4) can be written in the form of the superposition of the basic solution and small disturbances of 
the normal mode with the wavenumber $k$ and the growth rate $\omega$ :

$$
\left(\begin{array}{c}
u_{v} \\
w_{v} \\
p_{v} \\
c_{v} \\
c_{l} \\
h \\
J
\end{array}\right)=\left(\begin{array}{c}
0 \\
\bar{w}_{v} \\
\bar{p}_{v}(z) \\
\bar{c}_{v}(z) \\
\bar{c}_{l} \\
1 \\
\bar{J}
\end{array}\right)+\left(\begin{array}{c}
\widetilde{u}_{v}(z) \\
\widetilde{w}_{v}(z) \\
\widetilde{p}_{v}(z) \\
\widetilde{c}_{v}(z) \\
\widetilde{c}_{l}(z) \\
\widetilde{h} \\
\widetilde{J}
\end{array}\right) \mathrm{e}^{\mathrm{i} k x+\omega t}
$$

Note that the film thickness and interface concentration at the basic state are time-dependent owing to condensation. As a consequence, the exponent of the disturbances in Eq. (7) does not have an exact linear dependency on time; in other words $\omega$ is a function of time, which is written in the form of integration of some function of the basic solution (see Refs. [7, 10]). However, for simplicity we consider only the instantaneous growth rate at a given time or film thickness and regard $\omega$ as constant in time in the analysis below (the frozen-time approach). We substitute Eq. (7) into Eqs. (1) and (4) and linearize the resulting equations with respect to the amplitudes of the disturbances (denoted by a tilde). Then Eqs. (1) become

$$
\begin{gathered}
\mathrm{i} k \widetilde{u}_{v}+\widetilde{w}_{v}^{\prime}=0, \\
\omega \widetilde{u}_{v}+\bar{w}_{v} \widetilde{u}_{v}^{\prime}=-\mathrm{i} k \widetilde{p}_{v}+\nu\left(-k^{2} \widetilde{u}_{v}+\widetilde{u}_{v}^{\prime \prime}\right), \\
\omega \widetilde{w}_{v}+\bar{w}_{v} \widetilde{w}_{v}^{\prime}=-\widetilde{p}_{v}+\nu\left(-k^{2} \widetilde{w}_{v}+\widetilde{w}_{v}^{\prime \prime}\right), \\
\Sigma_{v}\left(\omega \widetilde{c}_{v}+\bar{w}_{v} \widetilde{c}_{v}+\bar{c}_{v}^{\prime} \widetilde{w}_{v}\right)=\nu\left(-k^{2} \widetilde{c}_{v}+\widetilde{c}_{v}^{\prime \prime}\right), \\
\omega \widetilde{h}=-\frac{S}{3} k^{4} \widetilde{h}+\frac{M a}{2} k^{2}(\widetilde{J}+\bar{J} \widetilde{h})+\Gamma \widetilde{J} . \\
\Sigma_{l} \omega \widetilde{c}_{l}=-k^{2} \widetilde{c}_{l}+\widetilde{c}_{l}^{\prime \prime}
\end{gathered}
$$

It should be noted that since the vapor concentration gradient exists in the basic state, the vapor concentration at the interface in boundary conditions (4) must be perturbed as

$$
c_{v}(h)=\bar{c}_{v I}+\left\{\widetilde{c}_{v}(1)+\bar{c}_{v}^{\prime}(1) \widetilde{h}\right\} \mathrm{e}^{\mathrm{i} k x+\omega t}
$$


Thus boundary conditions (4) are linearized as follows:

$$
\begin{gathered}
\widetilde{u}_{v}^{\prime}(1+\Delta)+\mathrm{i} k \widetilde{w}_{v}(1+\Delta)=0, \quad \widetilde{p}_{v}(1+\Delta)=0, \quad \widetilde{c}_{v}(1+\Delta)=0 \\
\widetilde{c}_{l}(0)=0 \\
-\nu \widetilde{J}=\Sigma_{v} \widetilde{w}_{v}(1) \\
\left.\widetilde{c}_{v}(1)-\widetilde{c}_{l}(1)+\bar{c}_{v}^{\prime \prime}(1) \widetilde{h}+\bar{J}_{\{} \widetilde{c}_{v}(1)+\bar{c}_{v}^{\prime}(1) \widetilde{h}-\widetilde{c}_{l}(1)\right\}+\left(\bar{c}_{v I}-\bar{c}_{l I}\right) \widetilde{J}=0 \\
\widetilde{u}_{l}(1)=\widetilde{u}_{v}(1)+\mathrm{i} k \bar{w}_{v} \widetilde{h} \\
\widetilde{c}_{l}(1)=c_{l}^{\prime}(\widetilde{J}+\bar{J} \widetilde{h}) \\
\widetilde{c}_{v}(1)+\bar{c}_{v}^{\prime}(1) \widetilde{h}=c_{v}^{\prime}(\widetilde{J}+\bar{J} \widetilde{h})
\end{gathered}
$$

where (see Ref. [12])

$$
\widetilde{u}_{l}(1)=\mathrm{i} k\left\{-\frac{S}{2} k^{2} \widetilde{h}+M a(\widetilde{J}+\bar{J} \widetilde{h})\right\} .
$$

Note that the terms proportional to $\widetilde{h}$ in Eq. (10d) cancel according to Eq. (6b).

\section{Solution for neutral stability}

Eqs. (8) and boundary conditions (10) constitute an eigenvalue problem for the growth rate. In order to solve this problem, we first rewrite Eq. (10g) as

$$
\frac{\widetilde{J}}{\widetilde{h}}=\frac{c_{v}^{\prime} \bar{J}-\bar{c}_{v}^{\prime}(1)}{f-c_{v}^{\prime}},
$$

where

$$
f=\frac{\widetilde{c}_{v}(1)}{\widetilde{J}}=-\frac{\nu \widetilde{c}_{v}(1)}{\Sigma_{v} \widetilde{w}_{v}(1)},
$$

using Eq. (10c). Here $f^{-1}$ signifies the nondimensionalized mass transfer coefficient (Sherwood number), because it represents the proportionality coefficient between the mass flux and concentration. Substitution of Eqs. (12) and (5b) into Eq. (8e) yields

$$
\omega=-\frac{S}{3} k^{4}+\frac{M a \bar{J}}{2} \frac{f+\bar{c}_{v I}-\bar{c}_{l I}}{f-c_{v}^{\prime}} k^{2}+\Gamma \bar{J} \frac{\bar{c}_{v I}-\bar{c}_{l I}+c_{v}^{\prime}}{f-c_{v}^{\prime}} .
$$

Here $f$ is a function of the wavenumber and growth rate. Therefore, once the functional form of $f$ is obtained, the growth rate should be calculated 
from Eq. (14). However, since the functional form of $f$ is complicated, it is difficult to directly solve Eq. (14) for $\omega$. For simplicity, we shall restrict our calculation to the case of neutral stability and set $\omega=0$ in the equations, focusing on stationary modes of instability. If the growth rate is purely imaginary (oscillatory modes), the corresponding solution is a traveling-wave one. We shall not consider such a solution because there is no physical reason to justify the existence of such a solution. Then the problem is reduced to obtaining the wavenumber dependence of $f$ by solving Eqs. (8) and (10). In expressing the solution, it is convenient to rescale the variables by the thickness of the gas boundary layer instead of the film thickness. We now introduce new variables for those depending on the latter as

$$
\begin{gathered}
\hat{z}=\frac{z-1}{\Delta}, \quad \hat{k}=k \Delta, \quad \hat{\boldsymbol{v}}=\boldsymbol{v} \Delta, \quad \hat{p}=p \Delta^{2}, \quad \hat{h}=\frac{h}{\Delta}, \\
\hat{J}=J \Delta, \quad \hat{S}=S \Delta, \quad \hat{M} a=M a \Delta, \quad \hat{f}=\frac{f}{\Delta},
\end{gathered}
$$

where we have omitted the overbar, tilde, and subscript, if they exist. Formally the variables with a hat correspond to those in whose scalings $d_{l}$ is replaced by $\delta$. Hereafter we shall drop the hat over the variables and the overbar of the basic mass flux and interface concentrations in the equations.

Noting that $\widetilde{u}_{v}$ can be eliminated by Eq. (8a), the equations which have to be solved are

$$
\begin{gathered}
\widetilde{p}_{v}^{\prime \prime}-k^{2} \widetilde{p}_{v}=0, \\
\widetilde{w}_{v}^{\prime \prime}+\frac{J}{\Sigma_{v}} \widetilde{w}_{v}^{\prime}-k^{2} \widetilde{w}_{v}=\frac{\widetilde{p}_{v}^{\prime}}{\nu} \\
\widetilde{c}_{v}^{\prime \prime}+J \widetilde{c}_{v}-k^{2} \widetilde{c}_{v}=-\left(c_{v I}-c_{l I}\right) \frac{\Sigma_{v} J}{\nu} \mathrm{e}^{-J z} \widetilde{w}_{v},
\end{gathered}
$$

for the bulk gas phase $(0<z<1)$. Here we have substituted Eqs. (5a) and (6) into Eqs. (8c) and (8d) to eliminate $\bar{w}_{v}$ and $\bar{c}_{v}^{\prime}$. For the liquid mass transport equation (8f), the long-wave approximation supposes that $\partial_{x} \ll \partial_{z}$ in the liquid phase. Then Eq. (8f) reduces to $\widetilde{c}_{l}^{\prime \prime}=0$ and we may regard $\widetilde{c}_{l}$ as constant from Eq. (10b). Similarly the boundary conditions become

$$
\begin{gathered}
\widetilde{w}_{v}^{\prime \prime}(1)+k^{2} \widetilde{w}_{v}(1)=0, \quad \widetilde{p}_{g}(1)=0, \quad \widetilde{c}_{v}(1)=0 \\
\widetilde{w}_{v}^{\prime}(0)=a \widetilde{w}_{v}(0)+b \widetilde{c}_{v}(0) \\
\widetilde{w}_{v}(0)=c \widetilde{c}_{v}(0)+d \widetilde{c}_{v}(0)
\end{gathered}
$$


where

$$
\begin{aligned}
a & =\frac{k^{2}}{c_{v I}-c_{l I}+c_{v}^{\prime}}\left\{\left(-\frac{S \Sigma_{v} k^{2}}{2 \nu J \Delta^{2}}+1\right) \frac{c_{v}^{\prime}}{\Delta}-\frac{M a \Sigma_{v}\left(c_{v I}-c_{l I}\right)}{\nu \Delta^{2}}\right\}, \\
b & =\frac{k^{2}}{c_{v I}-c_{l I}+c_{v}^{\prime}}\left\{-\frac{S k^{2}}{2 J \Delta^{2}}+\frac{M a}{\Delta}+\frac{\nu}{\Sigma_{v}}\right\}, \\
c & =\frac{c_{v I}-c_{l I}+c_{v}^{\prime}}{\left(c_{v I}-c_{l I}\right)\left(c_{v I}-c_{l I}+c_{v}^{\prime}-J c_{l}^{\prime}\right)} \frac{\nu}{\Sigma_{v}} \\
d & =\frac{c_{v I}-c_{l I}+c_{v}^{\prime}-c_{l}^{\prime}}{\left(c_{v I}-c_{l I}\right)\left(c_{v I}-c_{l I}+c_{v}^{\prime}-J c_{l}^{\prime}\right)} \frac{\nu J}{\Sigma_{v}}
\end{aligned}
$$

and we have eliminated $\widetilde{u}_{v}, \widetilde{J}, \widetilde{h}, \bar{w}_{v}$ and $\bar{c}_{v}^{\prime}(0)$ from Eq. (10) with the aid of Eqs. (5) and (8a). The solution of Eq. (16) subject to boundary conditions (17) is determined except for an unknown proportionality factor $C$ as follows:

$$
\begin{aligned}
\widetilde{p}_{v}= & \frac{C \nu J}{\Sigma_{v}\left(c_{v I}-c_{l I}\right)} A_{p} k \sinh \{k(z-1)\} \\
\widetilde{w}_{v}= & C \mathrm{e}^{-\frac{J z}{2 \Sigma_{v}}}\left[k_{1} \sinh \left\{k_{1}(z-1)\right\}+\frac{J}{2 \Sigma_{v}} \cosh \left\{k_{1}(z-1)\right\}\right]+\frac{\Sigma_{v} \widetilde{p}_{v}}{\nu J} \\
\widetilde{c}_{v}= & \frac{C \Sigma_{v}}{\nu}\left[\mathrm{e}^{-\left(1+\frac{1}{2 \Sigma_{v}}\right) J z}\left\{A_{1} \sinh \left(k_{1}(z-1)\right)+A_{2} \cosh \left(k_{1}(z-1)\right)\right\}\right. \\
& +\mathrm{e}^{-\frac{J}{2} z}\left\{A_{3} \sinh \left(k_{2}(z-1)\right)+A_{4} \cosh \left(k_{2}(z-1)\right)\right\} \\
& \left.+A_{p} \mathrm{e}^{-J z} \cosh (k(z-1))\right]
\end{aligned}
$$


where

$$
\begin{aligned}
& k_{1}=\sqrt{\left(\frac{J}{2 \Sigma_{v}}\right)^{2}+k^{2}}, \quad k_{2}=\sqrt{\left(\frac{J}{2}\right)^{2}+k^{2}}, \\
& \left(\begin{array}{l}
A_{1} \\
A_{2}
\end{array}\right)=\frac{c_{v I}-c_{l I}}{\left(1+\Sigma_{v}\right) k^{2}}\left(\Sigma_{v}\left\{2\left(\frac{J}{2 \Sigma_{v}}\right)^{2}+k^{2}\right\}\right), \\
& \left(\begin{array}{l}
A_{p} \\
A_{3}
\end{array}\right)=\frac{1}{A_{11} A_{22}-A_{12} A_{21}}\left(\begin{array}{cc}
A_{11} & A_{12} \\
A_{21} & A_{22}
\end{array}\right)\left(\begin{array}{l}
B_{1} \\
B_{2}
\end{array}\right) \\
& A_{4}=-\mathrm{e}^{-\frac{J}{2}}\left(\mathrm{e}^{-\frac{J}{2 \Sigma_{v}}} A_{2}+A_{p}\right) \text {, } \\
& A_{11}=-\frac{\Sigma_{v} c}{\nu}\left(\frac{J}{2} \sinh k_{2}+k_{2} \cosh k_{2}\right)+\frac{\Sigma_{v} d}{\nu} \sinh k_{2}, \\
& A_{12}=-\frac{\Sigma_{v} b}{\nu} \sinh k_{2} \\
& A_{21}=-\frac{\Sigma_{v} c}{\nu}\left\{k \sinh k+J \cosh k-\mathrm{e}^{-\frac{J}{2}}\left(k_{2} \sinh k_{2}+\frac{J}{2} \cosh k_{2}\right)\right\} \\
& +\frac{\Sigma_{v} d}{\nu}\left(\cosh k-\mathrm{e}^{-\frac{J}{2}} \cosh k_{2}\right)+\frac{k \sinh k}{c_{v I}-c_{l I}}, \\
& A_{22}=\frac{k(k \cosh k+a \sinh k)}{c_{v I}-c_{l I}}-\frac{\Sigma_{v} b}{\nu}\left(\cosh k-\mathrm{e}^{-\frac{J}{2}} \cosh k_{2}\right), \\
& B_{1}=-k^{2} \cosh k_{1}+a\left(-k_{1} \sinh k_{1}+\frac{J}{2 \Sigma_{v}} \cosh k_{1}\right) \\
& +\frac{\Sigma_{v} b}{\nu}\left[-A_{1} \sinh k_{1}+A_{2}\left\{\cosh k_{1}-\mathrm{e}^{-\frac{J}{2}\left(1+\frac{1}{\Sigma_{v}}\right)} \cosh k_{2}\right\}\right], \\
& B_{2}=k_{1} \sinh k_{1}-\frac{J}{2 \Sigma_{v}} \cosh k_{1}-\frac{\Sigma_{v}^{2}\left(c_{v I}-c_{l I}\right) c}{\nu\left(1+\Sigma_{v}\right)}\left\{\left(1-\frac{J^{2}}{\Sigma_{v} k^{2}}\right) k_{1} \sinh k_{1}\right. \\
& \left.+\left(1-\frac{1}{2 \Sigma_{v}}+\frac{J^{2}}{2 \Sigma_{v}^{2} k^{2}}\right) J \cosh k_{1}\right\} \\
& +\frac{\Sigma_{v} c A_{2}}{\nu} \mathrm{e}^{-\frac{J}{2}\left(1+\frac{1}{\Sigma_{v}}\right)}\left(k_{2} \sinh k_{2}+\frac{J}{2} \cosh k_{2}\right) \\
& +\frac{\Sigma_{v} d}{\nu}\left[-A_{1} \sinh k_{1}+A_{2}\left\{\cosh k_{1}-\mathrm{e}^{-\frac{J}{2}\left(1+\frac{1}{\Sigma_{v}}\right)} \cosh k_{2}\right\}\right] .
\end{aligned}
$$

Since $C$ in the solution (19) cancels in the definition of $f(13)$, the value 


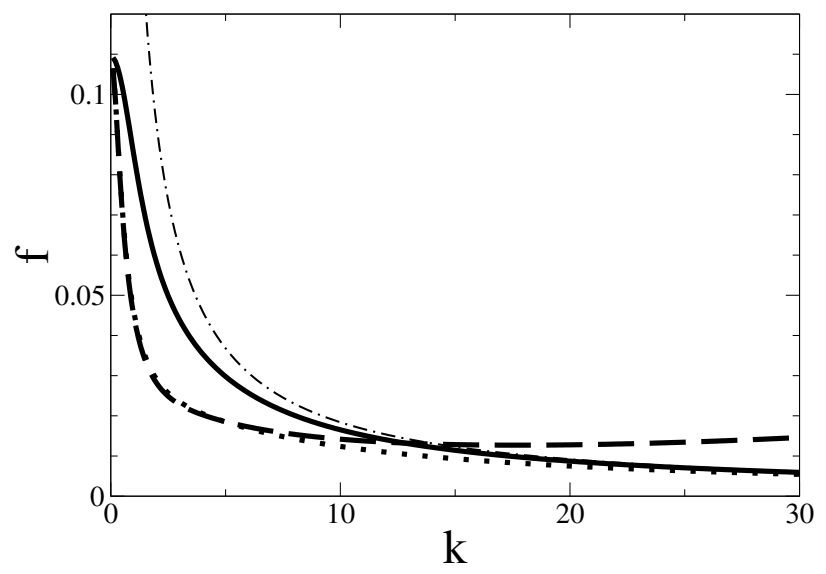

Figure 2: The wavenumber dependence of the function $f$ for the water-ethanol system at atmospheric pressure. The solid, dashed, and dotted curves are for $d_{l}=1 \mu \mathrm{m}$ and $\delta=1 \mathrm{~mm}, d_{l}=10 \mu \mathrm{m}$ and $\delta=1 \mathrm{~mm}$, and $d_{l}=10 \mu \mathrm{m}$ and $\delta=1 \mathrm{~cm}$, respectively. The dot-dashed one indicates $\nu /\left(\Sigma_{v} c k\right)$. Here we have set $c_{0}=0.1$ and $T_{r}=370 \mathrm{~K}$.

of $f$ is obtained from the solution (19) with Eq. (20). It can be shown that

$$
\lim _{k \rightarrow 0} f=\left(c_{v I}-c_{l I}\right) \frac{c_{v I}-c_{l I}+c_{v}^{\prime}-c_{l}^{\prime}\left(\mathrm{e}^{J}-1\right)}{c_{v I}-c_{l I}+c_{v}^{\prime}+c_{l}^{\prime}\left(\mathrm{e}^{J}-1\right)} .
$$

This value depends on $J$ or $c_{0}$. If $J$ is sufficiently small, $f$ is positive. However, $f$ becomes negative for large $J$. Note that $f$ is dependent on $\Delta$ through $a$ and $b$ in Eq. (18). Clearly this dependence disappears in the limit $d_{l} \rightarrow 0$ or $\Delta \rightarrow \infty$. Fig. 2 shows the value of $f$ as a function of the wavenumber for various liquid and gas thicknesses. Here we have used the physical properties for the water-ethanol system, which have been calculated according to Refs. $[3,7,14]$. For simplicity, the temperature and concentration in the parameters have been evaluated at the interface. Although not plotted, the curve for the limit $d_{l} \rightarrow 0(\Delta \rightarrow \infty)$ almost coincides with that for $d_{l}=1 \mu \mathrm{m}$ and $\delta=1 \mathrm{~mm}$ in the wavenumber region shown in Fig. 2. If we set $d_{l}=10 \mu \mathrm{m}$, the curve for $\delta=1 \mathrm{~mm}$ deviates from that for $d_{l}=1 \mu \mathrm{m}$. However, the curve for $d_{l}=10 \mu \mathrm{m}$ and $\delta=1 \mathrm{~cm}$ again approaches the latter for larger wavenumbers because $\Delta$ becomes large. 
According to the rescalings (15), Eq. (14) is written as

$$
0=-\frac{S}{3} k^{4}+\frac{M a J}{2} \frac{f+\frac{c_{v I}-c_{l I}}{\Delta}}{f-\frac{c_{v}^{\prime}}{\Delta}} \Delta k^{2}+\Gamma J \Delta^{3} \frac{c_{v I}-c_{l I}+c_{v}^{\prime}}{f-\frac{c_{v}^{\prime}}{\Delta}} .
$$

For the water-ethanol system, $c_{v}^{\prime}<-3$ unless the ethanol concentration sufficiently approaches 1 , where $c_{v}^{\prime}$ becomes positive. If $f>-\left(c_{v I}-c_{l I}\right) / \Delta$, the Marangoni and mass gain terms in Eq. (22) are positive and negative, which means that the Marangoni and mass gain effects are destabilizing and stabilizing.

Eq. (22) represents the neutral stability curve in the $k$ - $\Delta$ plane. Since all of the parameters have been normalized by the gas boundary layer thickness as in Eq. (15), we shall fix the latter in the following analysis. Then varying $\Delta$ corresponds to varying the film thickness. Therefore a film thickness of neutral stability is determined from Eq. (22) for each $k$. In Eq. (22), $f$ is generally a complicated function of $\Delta$. However, as mentioned above, the $\Delta$ dependence of $f$ is negligible for very thin films. Furthermore, mass gain is typically effective only for a few micrometers' thicknesses (or below). For these reasons, we shall set $\Delta^{-1}=0$ in $f$ when calculating the neutral stability curve from Eq. (22). In Fig. 3, the neutral stability curve is depicted in a dimensional wavenumber versus film thickness plane for the water-ethanol system. Here we stress that it is the wall temperature, not the interface temperature, that is directly controlled in experiments. However, as mentioned after Eq. ( $4 \mathrm{~g})$ we have fixed the value of the latter as the reference one throughout the calculation. Fig. 3 demonstrates the existence of a critical thickness below which the flat film is stable due to the mass gain effect.

In the analysis so far, we have dealt with the growth rate of disturbances irrelevant to that of the basic film thickness itself. Nevertheless, in order for the inhomogeneity of the condensate to appear the former has to exceed the latter. Since the latter is expressed as $\Gamma J / \Delta$ from Eq. (1d), the above condition can be written as follows:

$$
-\frac{S}{3} k^{4}+\frac{M a J}{2} \frac{f+\frac{c_{v I}-c_{l I}}{\Delta}}{f-\frac{c_{v}^{\prime}}{\Delta}} \Delta k^{2}+\Gamma J \Delta^{3} \frac{c_{v I}-c_{l I}+c_{v}^{\prime}}{f-\frac{c_{v}^{\prime}}{\Delta}}>\Gamma J \Delta^{4} .
$$

If the left and right hand sides of Eq. (23) are equal, disturbances are neutrally stable relative to the increase of the average film thickness. Thus the 


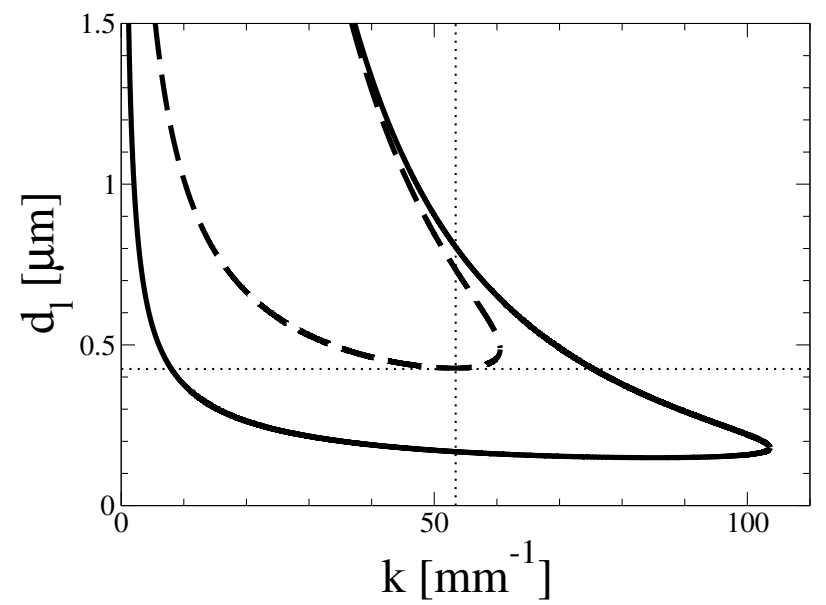

Figure 3: The neutral stability curve obtained from Eq. (22) (solid) and Eq. (23) (dashed) in a dimensional wavenumber versus film thickness plane for the water-ethanol system at atmospheric pressure. $\delta=1 \mathrm{~mm}, c_{0}=0.1$ and $T_{r}=370 \mathrm{~K}$. Here we have set $\Delta^{-1}=0$ in $f$. The dotted lines represent the critical thickness and wavenumber numerically calculated from Eq. (23) with the asymptotic form of $f(24)$.

relative neutral stability is defined. The relative neutral stability curve is also drawn in Fig. 3. Note that since the growth rate is no longer zero in Eq. (23) the value of $f$ is slightly varied from that for $\omega=0$. However, for simplicity we have neglected this dependence of $f$ on the growth rate in the calculation of the relative neutral stability curve. From Fig. 3 it is found that the unstable domain significantly shrinks and the critical thickness and its wavenumber change from the original ones.

\section{Asymptotic analysis for large wavenumbers}

If $\Delta^{-1}=0, f$ has a following asymptotic form for large wavenumbers:

$$
f \sim \frac{\nu}{\Sigma_{v} c k}
$$

In Fig. 2, we have also plotted this asymptotic form by the dot-dashed curve. A good agreement with the curve for $d_{l}=1 \mu \mathrm{m}$ and $\delta=1 \mathrm{~mm}$ is achieved for $k>20$. Comparing Figs. 2 and 3, one finds that the critical thickness takes place within this asymptotic regime. Therefore, an asymptotic approach is 

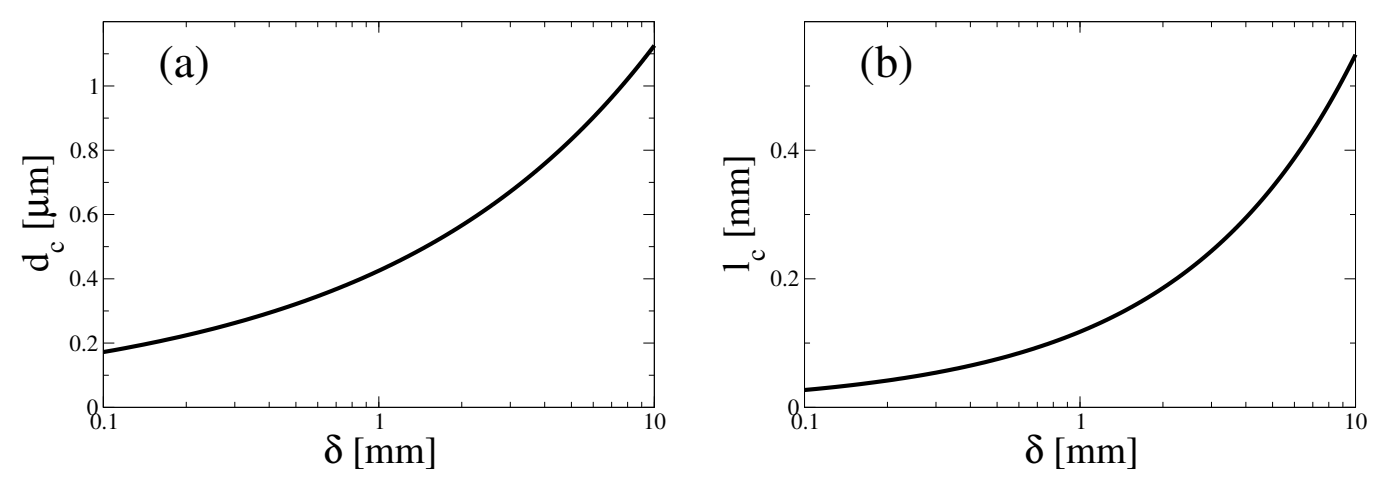

Figure 4: The dependence on the boundary layer thickness of the critical thickness $d_{c}$ (a) and wavelength $l_{c}$ (b) numerically calculated from Eq. (23) with the asymptotic form of $f$ (24) for the water-ethanol system at atmospheric pressure. $c_{0}=0.1$ and $T_{r}=370 \mathrm{~K}$.

applicable to Eq. (23) for obtaining the critical thickness and wavenumber. The critical thickness and wavenumber numerically calculated from Eq. (23) with the asymptotic form of $f(24)$ are indicated by the dotted lines in Fig. 3 and found to quite well agree with those of the neutral stability curve obtained without using the asymptotic approach.

Note that the critical thickness and wavelength depend on the boundary layer thickness. Their dependence is plotted in Fig. 4 employing the asymptotic form of $f(24)$. Both increase with the boundary layer thickness. In experiments, the increase of the vapor velocity above the condensate leads to the decrease of the boundary layer thickness.

Fig. 5 shows the dependence on the temperature of the critical thickness and wavenumber for various ambient vapor concentrations. Since the asymptotic form (24) is valid for $\Delta^{-1} \sim 0$, the lines are truncated so that the critical thickness becomes less than $1 \mu \mathrm{m}$. The divergence of the critical thickness around the vapor and liquid line temperatures can be explained as follows. In the former case the condensate mass flux is small, so that the Marangoni effect is weak. In the latter $c^{-1}$ decreases with larger mass fluxes from Eq. (18c) (note that $c_{l}^{\prime}<0$ ). Then the inverse Sherwood number $f$ also decreases from Eq. (24) and thereby the Marangoni term in Eq. (23) becomes smaller. The critical wavelength is generally smaller for smaller critical thicknesses and the minimum point of the former almost corresponds to that of the latter. This relationship between the critical wavelength and thickness is obvious given that the effect of surface tension becomes stronger for thicker films as shown in Eq. (23), which suppresses larger-wavenumber disturbances. 

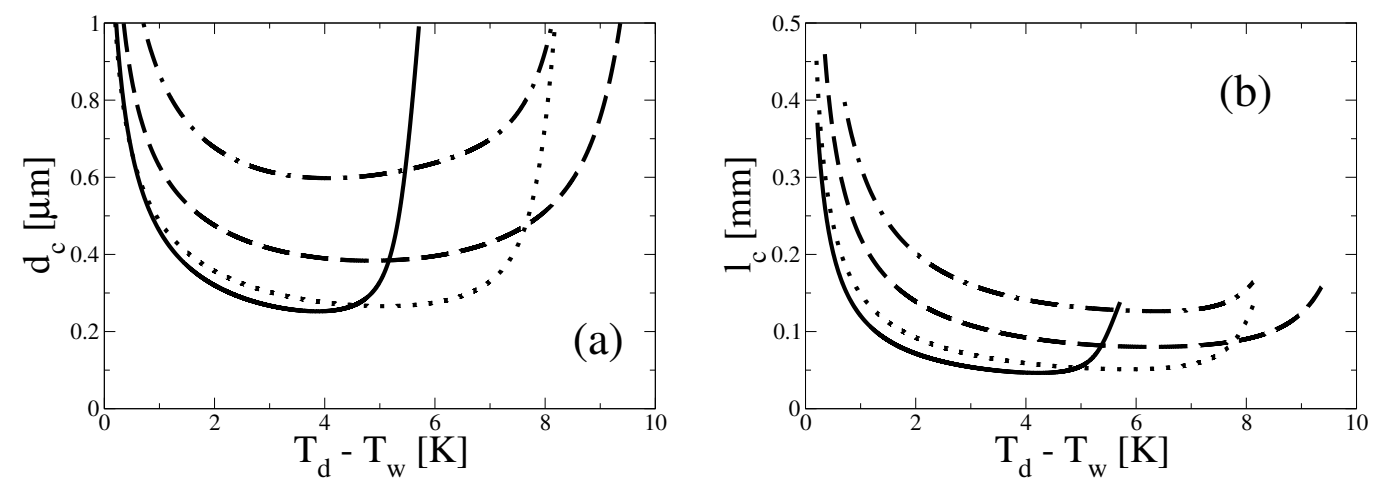

Figure 5: The dependence on the temperature of the critical thickness $d_{c}$ (a) and wavelength $l_{c}$ (b) numerically calculated from Eq. (23) with the asymptotic form of $f(24)$ for the water-ethanol system at atmospheric pressure. $c_{0}=0.07,0.17,0.37$ and 0.52 for the solid, dotted, dashed and dot-dashed curves, respectively. Here $T_{d}$ and $T_{w}$ are the dew point (vapor line) temperature for each vapor concentration and wall temperature. $\delta=0.5 \mathrm{~mm}$. The critical wavelength is plotted in the region where the corresponding critical thickness is less than $1 \mu \mathrm{m}$.

According to the experimental results [5] the initial drop distance exhibits a U-shaped curve against the surface subcooling and its minimum value is about $30-150 \mu \mathrm{m}$ with a larger value for larger ethanol mass fractions. This behavior of the initial drop distance toward the temperature and concentration can be seen in the critical wavelength presented in Fig. 5 (b). Moreover, in Fig. 5 we have suitably adjusted the boundary layer thickness so that the critical wavelength quantitatively agrees with the experimental data of the initial drop distance [5], aside from the value of the surface subcooling. Note that, however, the critical wavelength obtained from the linear theory does not necessarily coincide with the initial drop distance because the maximum growth wavenumber varies with the film thickness.

\section{Conclusions}

We have investigated the linear stability of a condensing thin liquid film of a binary vapor mixture by solving directly the bulk equations of the gas phase. As in the previous studies [7, 13], the boundary layer of a finite thickness has been introduced above the liquid film, within which the variables are disturbed against the quasi-steady basic state. The dynamics of the liquid film is described by the long-wave equation $[7,11]$. The dispersion relation is expressed in terms of the function $f$ in Eq. (13), whose reciprocal corresponds 
to the nondimensionalized mass transfer coefficient (Sherwood number). In our analysis the case of neutral stability has been examined by setting the growth rate to zero. In order to determine the functional form of $f$, we have solved directly the linearized governing equations for the amplitudes of disturbances (16) together with the boundary conditions (17).

The neutral stability condition is described by Eq. (22) as a function of $\Delta$, which represents the inverse film thickness under the constant boundary layer thickness. The neutral stability curve has been plotted in the wavenumber versus film thickness plane for the water-ethanol system. It has been found that the flat film becomes stable below a certain thickness due to the mass gain effect. However, if we consider the thickening of the liquid film by condensation, the relative neutral stability can be defined such that the growth rate of a disturbance is equal to that of the basic film thickness, which is represented by Eq. (23). The critical thickness and wavenumber obtained from Eq. (23) significantly change from those obtained from Eq. (22), as shown in Fig. 3.

For large wavenumbers $f$ has the asymptotic form (24). Employing this asymptotic form the critical thickness and wavelength were numerically calculated. We have examined their dependence on the boundary layer thickness, temperature and ambient vapor concentration. The critical thickness and wavelength increase with the boundary layer thickness. The critical wavelength predicted by our theory and the initial drop distance observed in the experiment [5] have the same trend in the temperature and concentration. In addition, we can also obtain their quantitative agreement by suitably adjusting the boundary layer thickness.

\section{Acknowledgments}

The author gratefully acknowledges Yoshio Utaka and the reviewer for their useful comments on this work.

\section{Appendix A. Influence of buoyancy due to the vapor concentration gradient}

In the analysis of the main text, buoyancy in the gas phase was not considered. However, if the molecular masses of the two species of the binary mixture differ, buoyancy is induced by the concentration gradient in the gas. In this appendix, we investigate an influence of buoyancy on the stability 
of the liquid film. In order to do this, the Boussinesq approximation is introduced, according to which the gas density of the buoyancy term in the Navier-Stokes equation (1b) is linearized as

$$
\rho_{v}=\rho_{v 0}\left\{1-\alpha\left(T_{v}-T_{0}\right)-\varepsilon\left(c_{v}-c_{0}\right)\right\}
$$

whereas the density variation is neglected elsewhere. Here $\alpha$ and $\varepsilon$ are the thermal and solutal expansion coefficients and $T_{v}$ and $T_{0}$ are the vapor and ambient temperatures. If the gas mixture may be regarded as an ideal gas, the gas density is expressed by

$$
\rho_{v}=\frac{M_{1} M_{2} p_{0}}{\left\{M_{1}-\left(M_{1}-M_{2}\right) c_{v}\right\} R T_{v}},
$$

where $M_{1}$ and $M_{2}$ are the molar masses of the components having lower and higher boiling points, $p_{0}$ is the total pressure of the gas and $R$ is the universal gas constant. Then the thermal and solutal expansion coefficients are calculated as

$$
\begin{aligned}
\alpha & =-\left.\frac{1}{\rho_{v 0}} \frac{\partial \rho_{v}}{\partial T_{v}}\right|_{T_{v}=T_{0}, c_{v}=c_{0}}=\frac{1}{T_{0}} \\
\varepsilon & =-\left.\frac{1}{\rho_{v 0}} \frac{\partial \rho_{v}}{\partial c_{v}}\right|_{T_{v}=T_{0}, c_{v}=c_{0}}=-\frac{M_{1}-M_{2}}{M_{1}-\left(M_{1}-M_{2}\right) c_{0}} .
\end{aligned}
$$

For the water-ethanol system, $|\varepsilon|>0.6, T_{0}>350 \mathrm{~K}$ and $\mid\left(T_{v}-T_{0}\right) /\left(c_{v}-\right.$ $\left.c_{0}\right) \mid \sim 20 \mathrm{~K}$ if we assume that the ambient vapor is at the coexistence state. Therefore we may neglect the temperature dependence of the density in this study. Then the buoyancy term is incorporated into the $z$-component of Eq. (1b) to give

$$
\partial_{t} w_{v}+\boldsymbol{v}_{v} \cdot \nabla w_{v}=-\partial_{z} p_{v}+\nu \nabla^{2} w_{v}-G+G \varepsilon\left(c_{v}-c_{0}\right)
$$

Accordingly the linearized equation (8c) is modified as

$$
\omega \widetilde{w}_{v}+\bar{w}_{v} \widetilde{w}_{v}^{\prime}=-\widetilde{p}_{v}+\nu\left(-k^{2} \widetilde{w}_{v}+\widetilde{w}_{v}^{\prime \prime}\right)+G \varepsilon \widetilde{c}_{v}
$$

In this case the solution is sought with the continuation technique using the software AUTO-07p [15]. The solutal expansion coefficient being a free parameter, the continuation is initiated from the solution for $\varepsilon=0$ (19) with the wavenumber fixed. After $\varepsilon$ reaches the corresponding value 


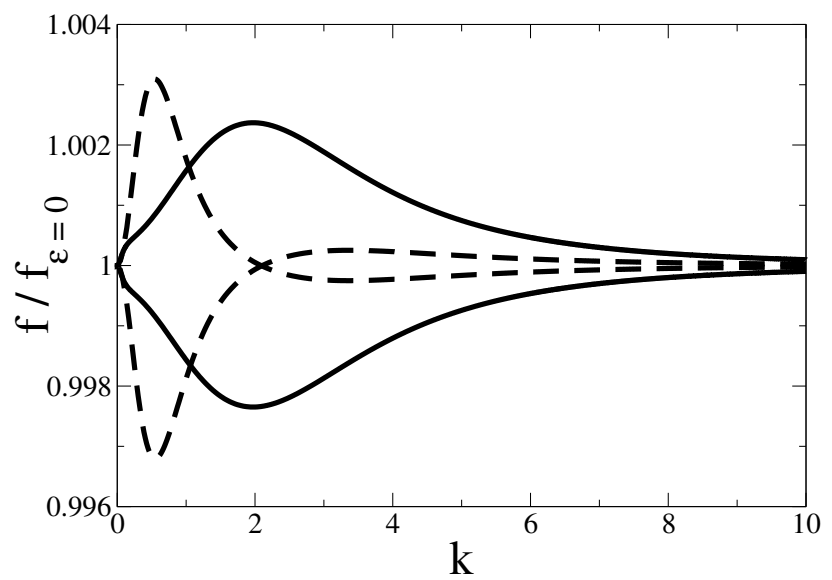

Figure A.6: The wavenumber dependence of the function $f / f_{\varepsilon=0}$ for the water-ethanol system at atmospheric pressure. The solid curves are for $T_{r}=365 \mathrm{~K}$ and $c_{0}=0.1$ and the dashed ones are for $T_{r}=360 \mathrm{~K}$ and $c_{0}=0.4$. The lower and upper curves (at larger wavenumbers for the dashed ones) correspond to the cases where gravity acts toward the liquid and gas. Here we have set $\Delta^{-1}=0$ in $f$.

for the water-ethanol system, the solution is then continued with respect to the wavenumber. The integral constraint $\int_{0}^{1} \widetilde{w}_{v} d z=1$ is imposed so as to fix the proportionality coefficient of the solution. Fig. A.6 shows the ratio of $f$ obtained from the continuation to that for $\varepsilon=0$ as a function of the wavenumber for the water-ethanol system. Here we have presented the results also for the case where gravity acts toward the vapor (i.e. pendant films). This case can be also interpreted as that where the lower-boilingpoint component of the mixture is lighter than the higher-boiling-point one, because in this case the sign of $\varepsilon$ oppositely changes (see Eq. (A.4)). The curves for the two cases are almost symmetric about $f / f_{\varepsilon=0}=1$. All of the curves tend to 1 in the limits $k \rightarrow 0$ and $k \rightarrow \infty$, where the influence of buoyancy disappears. Here we have set the interface temperatures relatively lower because the concentration difference across the boundary layer is larger for lower temperatures with the fixed value of the ambient vapor concentration. The variation of $f$ due to buoyancy is very small (within $0.4 \%$ ). Therefore, there is little influence of buoyancy on the stability of the liquid film. 


\section{References}

[1] V. V. Mirkovich, R. W. Missen, Non-filmwise condensation of binary vapors of miscible liquids, Canadian J. Chem. Eng. 39 (1961) 86-87.

[2] J. D. Ford, R. W. Missen, On the conditions for stability of falling films subject to surface tension disturbances; the condensation of binary vapor, Canadian J. Chem. Eng. 46 (1968) 309-312.

[3] Y. Li, J. Yan, J. Wang, G. Wang, A Semi-Empirical Model for Condensation Heat Transfer Coefficient of Mixed Ethanol-Water Vapors, J. Heat Transfer 133 (2011) 061501-1—061501-11.

[4] X. Ma, Z. Lan, W. Xu, M. Wang, S. Wang, Effect of surface free energy difference on steam-ethanol mixture condensation heat transfer, Int. J. Heat Mass Transfer 55 (2012) 531-537.

[5] Y. Utaka, T. Kenmotsu, S. Yokoyama, A Study on Marangoni Condensation (Measurement and Observation for Water and Ethanol Vapor Mixture), in: Proceedings of 11th International Heat Transfer Conference, 6 (1998), pp. 397-402.

[6] K. Hijikata, Y. Fukasaku, O. Nakabeppu, Theoretical and Experimental Studies on the Pseudo-Dropwise Condensation of a Binary Vapor Mixture, J. Heat Transfer 118 (1996) 140-147.

[7] K. Kanatani, Stability of a condensing liquid film in a binary vapor mixture system, Int. J. Heat Mass Transfer 58 (2013) 413-419.

[8] E. Sultan, A. Boudaoud, M. Ben Amar, Diffusion-limited evaporation of thin polar liquid films, J. Eng. Math. 50 (2004) 209-222.

[9] E. Sultan, A. Boudaoud, M. Ben Amar, Evaporation of a thin film: diffusion of the vapour and Marangoni instabilities, J. Fluid Mech. 543 (2005) 183-202.

[10] J. P. Burelbach, S. G. Bankoff, S. H. Davis, Nonlinear stability of evaporating/condensing liquid films, J. Fluid Mech. 195 (1988) 463-494.

[11] A. Oron, S. H. Davis, S. G. Bankoff, Long-scale evolution of thin liquid films, Rev. Mod. Phys. 69 (1997) 931-980. 
[12] K. Kanatani, Effects of convection and diffusion of the vapour in evaporating liquid films, J. Fluid Mech. 732 (2013) 128-149.

[13] B. Haut, P. Colinet, Surface-tension-driven instabilities of a pure liquid layer evaporating into an inert gas, J. Colloid Interface Sci. 285 (2005) 296-305.

[14] T. Fujii, Theory of Laminar Film Condensation, Springer-Verlag, New York, 1991.

[15] E. J. Doedel, A. R. Champneys, F. Dercole, T. Fairgrieve, Y. Kuznetsov, B. Oldeman, R. Paffenroth, B. Sanddtede, X. Wang, C. Zhang, AUTO07p: Continuation and Bifurcation Software for Ordinary Differential Equations, Concordia University, http://indy.cs.concordia.ca/auto/, 2007. 